\title{
Salinomycin induces calpain and cytochrome $c$-mediated neuronal cell death
}

\author{
W Boehmerle*,1,2 and M Endres ${ }^{1,2,3}$
}

Salinomycin is a polyether antibiotic with properties of an ionophore, which is commonly used as cocciodiostatic drug and has been shown to be highly effective in the elimination of cancer stem cells (CSCs) both in vitro and in vivo. One important caveat for the potential clinical application of salinomycin is its marked neural and muscular toxicity. In the present study we show that salinomycin in concentrations effective against CSCs exerts profound toxicity towards both dorsal root ganglia as well as Schwann cells. This toxic effect is mediated by elevated cytosolic $\mathrm{Na}^{+}$concentrations, which in turn cause an increase of cytosolic $\mathrm{Ca}^{2+}$ by means of $\mathrm{Na}^{+} / \mathrm{Ca}^{2+}$ exchangers (NCXs) in the plasma membrane as well as the mitochondria. Elevated $\mathrm{Ca}^{2+}$ then leads to calpain activation, which triggers caspase-dependent apoptosis involving caspases 12,9 and 3 . In addition, cytochrome $c$ released from depolarized mitochondria directly activates caspase 9 . Combined inhibition of calpain and the mitochondrial NCXs resulted in significantly decreased cytotoxicity and was comparable to caspase 3 inhibition. These findings improve our understanding of mechanisms involved in the pathogenesis of peripheral neuropathy and are important to devise strategies for the prevention of neurotoxic side effects induced by salinomycin.

Cell Death and Disease (2011) 2, e168; doi:10.1038/cddis.2011.46; published online 2 June 2011

Subject Category: Neuroscience

Salinomycin is a monocarboxylic polyether antibiotic that is produced by a Streptomyces albus strain and acts as an ionophore. ${ }^{1}$ It is widely used as an antiprotozoal agent against coccidial parasites, for example in poultry farming and as a growth promoter in chicken, pigs as well as ruminants. Its mechanism of action involves the formation of lipid-soluble complexes with cations, thereby facilitating bidirectional ion flux through lipid barriers by passive diffusion processes. ${ }^{1}$ There is increasing interest in the development of salinomycin as a potential clinical treatment for cancer, as it was shown in a high-throughput screen that salinomycin is a highly effective agent in the elimination of cancer stem cells (CSCs). ${ }^{2}$ In addition, there is emerging evidence that it may also sensitize cancer cells to cytostatic drugs such as doxorubicin. ${ }^{3}$ Established treatment strategies for various tumors kill the majority of tumor cells, but fail to remove CSCs, which then are thought to be responsible for local relapses and distant metastases. An effective therapy against this tumor cell population is therefore of great clinical significance.

One important caveat for the potential clinical use of salinomycin is its severe toxicity. A number of incidents occurred in the past, where the substance was accidentally fed or ingested in higher doses. ${ }^{4}$ One particularly notable episode occurred with salinomycin-contaminated cat food in the Netherlands in 1996. Poisoned cats developed a severe sensorimotor polyneuropathy with acute hind-limb paralysis, which in some cases progressed to tetraparalysis, respiratory failure and death. Post-mortem studies showed a combined, predominantly axonal but also demyelinating neuropathy. ${ }^{5}$ Peripheral nerve degeneration was also reported in dogs for the salinomycin derivative narasin. ${ }^{6}$ In a case of incidental human poisoning by salinomycin, rhabdomyolysis but also decreased reflexes suggestive of peripheral nerve lesions were reported. ${ }^{4}$

The precise mechanism of salinomycin-mediated neurotoxicity is not known. Interestingly, it was observed that salinomycin alters cytosolic calcium $\left(\mathrm{Ca}^{2+}\right)$ concentrations in rabbit hearts, ${ }^{7}$ and it was shown to inhibit mitochondrial function in isolated mitochondria. ${ }^{8}$ We therefore aimed to determine the effects of salinomycin on cell viability as well as the ional homeostasis of murine dorsal root ganglia neurons (DRGNs), and attempted to characterize the resulting downstream effector mechanisms. We found that salinomycin strongly reduces cell viability by means of calpain and cytochrome $c$ mediated caspase 9 and subsequent caspase 3 activation. Furthermore, we observed an increase of cytosolic $\mathrm{Na}^{+}$concentrations, which leads to an increase of cytosolic $\mathrm{Ca}^{2+}$ through the $\mathrm{Na}^{+} / \mathrm{Ca}^{2+}$ exchangers (NCXs) in the plasma membrane and mitochondria. Inhibition of calpain and the mitochondrial NCXs showed similar degrees of neuroprotection compared with caspase 3 inhibition.

\footnotetext{
${ }^{1}$ Department of Neurology, Charite-Universitätsmedizin Berlin, Berlin, Germany; ${ }^{2}$ Cluster of Excellence NeuroCure, Charite-Universitätsmedizin Berlin, Berlin, Germany and ${ }^{3}$ Center for Stroke Research Berlin, Charite-Universitätsmedizin Berlin, Berlin, Germany

${ }^{*}$ Corresponding author: W Boehmerle, Klinik für Neurologie, Charité-Universitätsmedizin Berlin, Chariteplatz 1, Berlin 10117, Germany. Tel: +49 30450560137 ; Fax: + 4930450560 932; E-mail: wolfgang.boehmerle@ charite.de

Keywords: polyneuropathy; dorsal root ganglia; sodium calcium exchanger; cancer stem cells; salinomycin

Abbreviations: $\mathrm{Ca}^{2+}$, calcium; ER, endoplasmic reticulum; $\mathrm{NCX}, \mathrm{Na}^{+} / \mathrm{Ca}^{2+}$ exchanger; DRGNs, dorsal root ganglia neurons; CSC, cancer stem cell; CGP, CGP-37157

Received 19.1.11; revised 05.4.11; accepted 21.4.11; Edited by A Verkhratsky
} 


\section{Results}

Prolonged exposure to salinomycin induces cell death in DRGNs. Salinomycin in the dose range of $1-10 \mu \mathrm{M}$ has been shown to be highly effective in the elimination of CSCs. ${ }^{2}$ We therefore tested whether these concentrations would impair cell viability in primary rat DRGNs. To discover early cellular alterations, we studied cells treated for $8 \mathrm{~h}$ and additionally assayed cell viability after $24 \mathrm{~h}$, as early changes in cell death have been shown to be potentially reversible (reviewed by Galluzzi et al. $^{9}$ ). A typical event of early apoptosis is the exposure of phosphatidylserine (PS) on the outer leaflet of the plasma membrane, which can be visualized by live-cell staining using fluorophore-conjugated annexin-V. DRGNs were treated for $8 \mathrm{~h}$ with $10 \mu \mathrm{M}$ salinomycin or vehicle (DMSO), and then labeled with annexin-V-enhanced green fluorescent protein (EGFP). Exposure to salinomycin led to a significantly higher number of annexin-V-positive DRGNs compared with control (41 $\pm 5 \%$; 749/6; versus $10 \pm 1 \%$; 785/6; $P=0.002$; Figure 1a). To further investigate whether prolonged exposure to salinomycin exerts cytotoxic effects in the low micromolar dose range, we investigated cell viability by means of a multiplexed assay measuring the activity of the mitochondrial dehydrogenase as well as protease activity released from dead cells. After incubation for $24 \mathrm{~h}$ with 1 and $10 \mu \mathrm{M}$ salinomycin or vehicle, cell viability was decreased to $27 \pm 1 \% \quad(n=9 ; \quad P<0.001)$ for $1 \mu \mathrm{M}$ and $15 \pm 2 \% \quad(n=9$; $P<0.001)$ for $10 \mu \mathrm{M}$ salinomycin compared with vehicle control (100 $\pm 7 \%, n=9$; Figure $1 \mathrm{~b}$ ). Taken together, these results show that salinomycin in micromolar concentrations exerts marked toxicity against peripheral neurons.

Effects of salinomycin on cytosolic $\mathrm{Na}^{+}$and $\mathrm{Ca}^{2+}$ concentrations in DRGNs. Given its ionic properties, we hypothesized that the observed neurotoxic effects are because of a disruption of the intracellular ion homeostasis. As salinomycin transports $\mathrm{Na}^{+}$more efficiently than $\mathrm{K}^{+},{ }^{1}$ we monitored cytosolic $\mathrm{Na}^{+}$changes in DRGNs using the ratiometric fluorescent $\mathrm{Na}^{+}$sensitive dye SBFI/AM. Addition of salinomycin but not vehicle lead to a rapid increase of the SBFI ratio, which reached a new steady state (Figure 2a). The average increase in SBFI ratio as compared with baseline, a measure that correlates to cytosolic $\mathrm{Na}^{+}$ concentrations, after addition of $10 \mu \mathrm{M}$ salinomycin was $0.41 \pm 0.03(29 / 5)$ and $0.03 \pm 0.01$ in vehicle-treated cells $(P<0.001 ; 33 / 5)$.

$\mathrm{Ca}^{2+}$ is an intracellular messenger that is involved in the regulation of many cellular functions, including cell death, and has been reported to be altered in the myocardium after salinomycin treatment. ${ }^{7}$ We thus hypothesized that a deranged $\mathrm{Ca}^{2+}$ homeostasis might be an important event in salinomycin-induced neurotoxicity. Cytosolic $\mathrm{Ca}^{2+}$ concentrations were measured in DRGNs loaded with the ratiometric $\mathrm{Ca}^{2+}$ indicator Fura-2/AM. Treatment with salinomycin lead to an average increase of $329 \pm 47 \mathrm{nM}$ in cytosolic $\mathrm{Ca}^{2+}(36 /$ $6)$ as compared with $10 \pm 2 \mathrm{nM}(36 / 6)$ in vehicle-treated cells $(P<0.05$; Figure $2 \mathrm{~b})$.

One possible explanation for the observed increase in cytosolic $\mathrm{Ca}^{2+}$ is that elevated cytosolic $\mathrm{Na}^{+}$concentrations cause a depolarization of the membrane potential, leading to a secondary $\mathrm{Ca}^{2+}$ influx through voltage-gated $\mathrm{Ca}^{2+}$ channels (VGCCs). We inhibited the VGCCs and most other $\mathrm{Ca}^{2+}$-permeable channels in the plasma membrane using $0.5 \mathrm{mM} \mathrm{CdCl}_{2}$ prior to salinomycin exposure, which however did not have a significant effect on the response amplitude (301 $\pm 33 \mathrm{nM}$; 36/6; Figure 2b). In order to determine the contribution of extracellular $\mathrm{Ca}^{2+}$ to this signal, cells were analyzed in a $\mathrm{Ca}^{2+}$-free solution. This pretreatment did not abolish the response, but the response amplitude was significantly decreased to $61 \pm 8 \mathrm{nM}$ (Figure $2 \mathrm{c}$; $P<0.05$; 36/6).
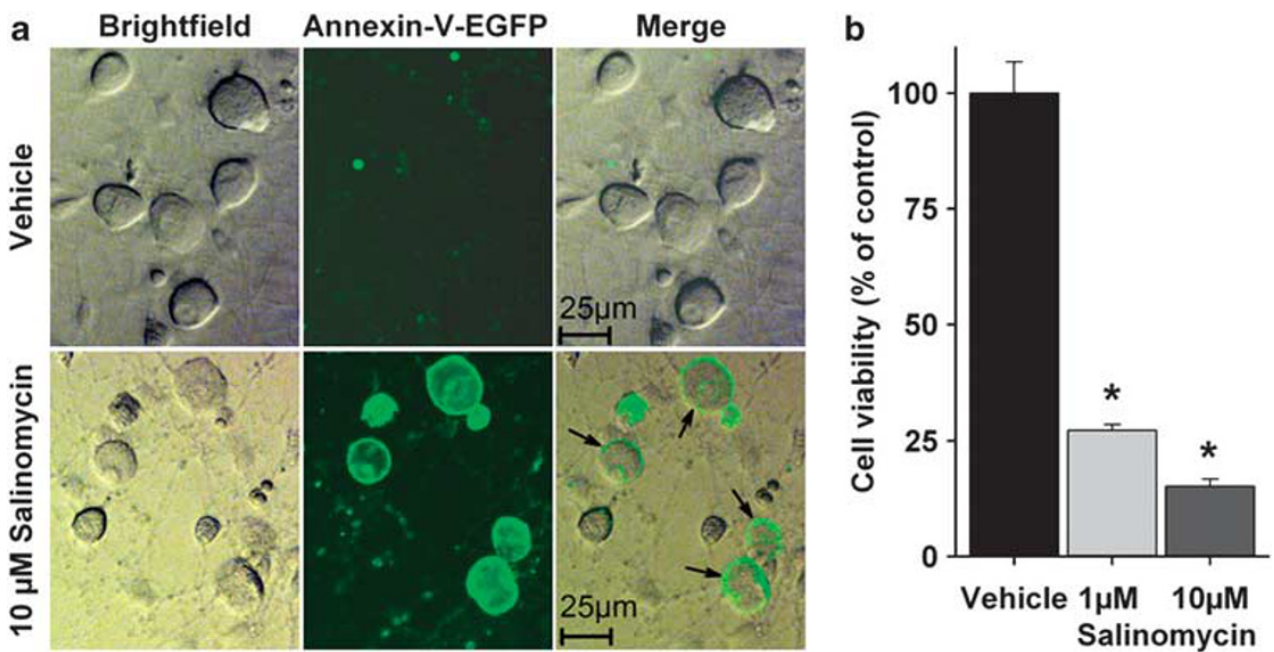

Figure 1 Salinomycin induces cell death in DRGNs. (a) Cells were exposed to $10 \mu \mathrm{M}$ salinomycin or vehicle for $8 \mathrm{~h}$ and stained with annexin-V-EGFP, a fluorescent marker for plasma membrane asymmetry. The vehicle-treated cells (upper panel) were mainly annexin-V-negative, whereas many salinomycin-treated cells (lower panel) showed a strong fluorescence signal (arrows). (b) DRGNs were treated with various concentrations of salinomycin or vehicle for $24 \mathrm{~h}$. Cell viability was measured with a multiplexed assay measuring the activity of the mitochondrial dehydrogenase as well as protease activity released from dead cells. Salinomycin treatment resulted in a marked reduction of cell viability. ${ }^{*} P<0.05$ 

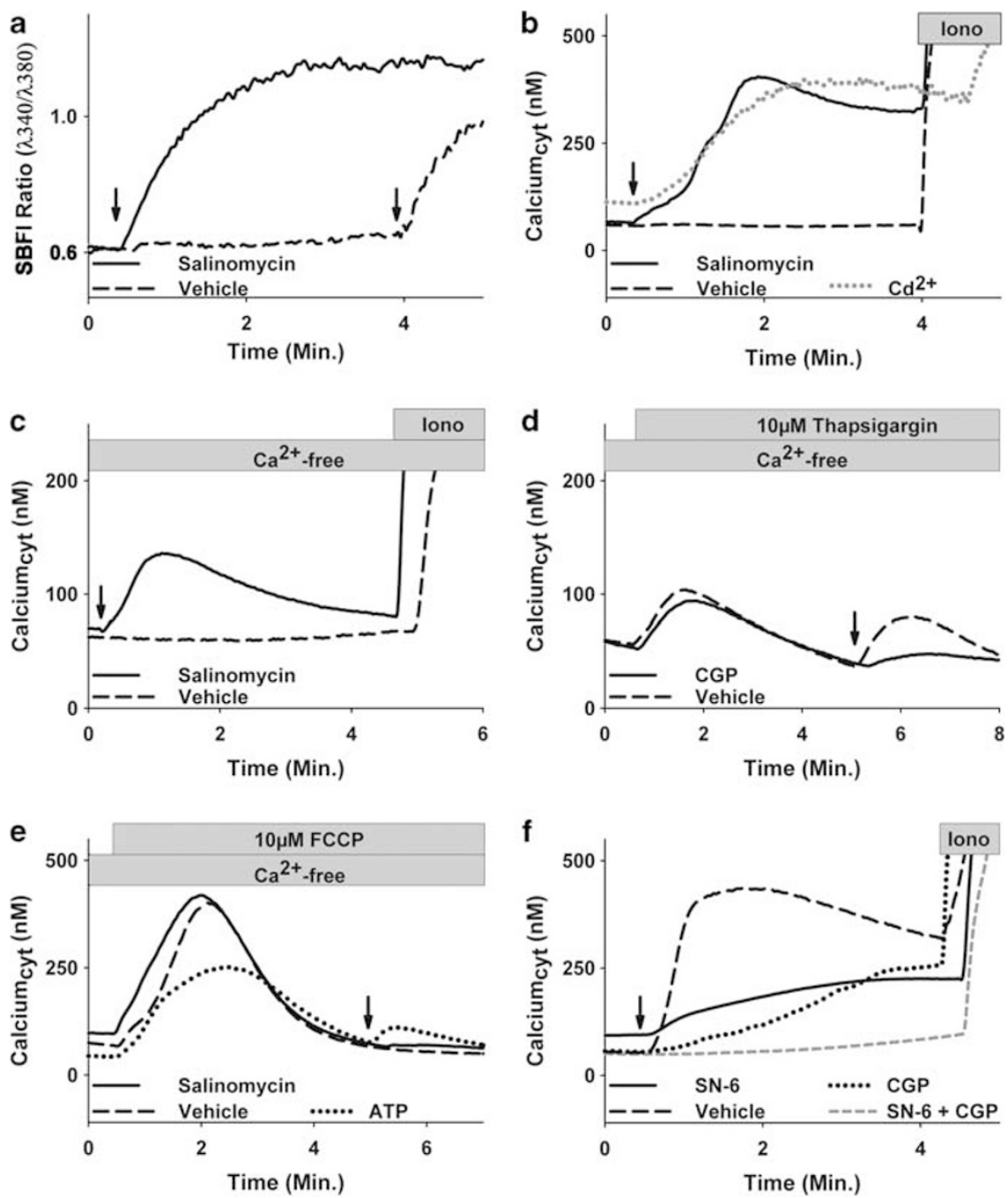

Figure 2 Salinomycin leads to increased cytosolic $\mathrm{Na}^{+}$and $\mathrm{Ca}^{2+}$ concentrations. (a) Salinomycin application (first arrow) leads to a rapid increase of cytosolic $\mathrm{Na}^{+}$ concentrations (solid line) in DRGNs. Vehicle treatment shows no effect (first arrow, dashed line) compared with later addition of salinomycin (second arrow, dashed line). (b) A representative $\mathrm{Ca}^{2+}$ response to treatment (arrow) with $10 \mu \mathrm{M}$ salinomycin (solid line) or vehicle (dashed line). Inhibition of VGCCs with $\mathrm{CdCl}_{2}$ does not affect the salinomycin-induced increase in cytosolic $\mathrm{Ca}^{2+}$ (gray dotted line). (c) In the absence of extracellular $\mathrm{Ca}^{2+}$, addition of salinomycin (arrow, solid line) but not vehicle (arrow, dashed line) leads to a transient increase in cytosolic $\mathrm{Ca}^{2+}$. (d) After depletion of $\mathrm{ER} \mathrm{Ca}^{2+}$ stores with thapsigargin, addition of salinomycin still elicits a response (arrow, dashed line, cells pretreated with vehicle). By contrast, pre-incubation with CGP, a selective inhibitor of mitochondrial NCXs, leads to a significantly reduced $\mathrm{Ca}^{2+}$ transient in response to salinomycin after thapsigargin treatment (arrow, solid line). (e) $\mathrm{Ca}^{2+}$ is released from mitochondrial stores in response to the mitochondrial uncoupler FCCP (first peak). Subsequent application (arrow) of salinomycin (solid line), vehicle (dashed line) or ATP (dotted line) shows an almost completely abrogated response to salinomycin but not ATP. (f) Pre-incubation with SN-6, an inhibitor of the NCXs in the plasma membrane (solid line), CGP (dotted line), or SN-6, and CGP combined (gray short-dashed line) leads to a significant reduction of the response amplitude compared with vehicle pre-incubation (dashed line) after application of $10 \mu \mathrm{M}$ salinomycin (arrow)

To further dissect the mechanism of salinomycin-induced increase in cytosolic $\mathrm{Ca}^{2+}$, we used thapsigargin, an inhibitor of the sarcoplasmic-endoplasmic reticulum (ER) $\mathrm{Ca}^{2+}$ ATPase, to deplete $\mathrm{Ca}^{2+}$ stored in the ER. After the initial $\mathrm{Ca}^{2+}$ release from the ER, the cytosolic $\mathrm{Ca}^{2+}$ concentrations returned to baseline levels, but addition of $10 \mu \mathrm{M}$ salinomycin still elicited a response of $30 \pm 3 \mathrm{nM}(33 / 5$; Figure $2 \mathrm{~d}$, dashed line). These results suggest that the initiation of the response to salinomycin does not depend on $\mathrm{Ca}^{2+}$ stored in the ER.

Another important buffer for $\mathrm{Ca}^{2+}$ ions are mitochondria. Addition of the mitochondrial-uncoupler FCCP $(10 \mu \mathrm{M})$ lead to a rapid release of mitochondrial $\mathrm{Ca}^{2+}$ (first peak in Figure 2e); further addition of $10 \mu \mathrm{M}$ salinomycin showed an almost completely abrogated response $(2 \pm 0.6 \mathrm{nM} ; P<0.05$ compared with both thapsigargin and vehicle pretreatment, 36/6; Figure 2e). By contrast, stimulation with $200 \mu \mathrm{M}$ ATP still elicited a response after FCCP pretreatment $(110 \pm 28 \mathrm{nM}$; $P<0.05$ compared with salinomycin, 26/6; Figure $2 e$ ).

Taken together, these results suggest that the increase of cytosolic $\mathrm{Ca}^{2+}$ induced by salinomycin is partly mediated by influx over the plasma membrane and partly by release of $\mathrm{Ca}^{2+}$ stored in mitochondria.

Salinomycin-induced $\mathrm{Ca}^{2+}$ changes are mediated by NCXs in the plasma membrane and mitochondria. In the presence of high levels of cytosolic $\mathrm{Na}^{+}$, the NCXs in the 
plasma membrane and mitochondria can go into a 'reverse mode' leading to accumulation of $\mathrm{Ca}^{2+}$ in the cytosol. In order to test this hypothesis, DRGNs were pre-incubated with CGP-37157 (CGP), which is a selective inhibitor of the mitochondrial NCXs. ${ }^{10}$ To avoid confounding $\mathrm{Ca}^{2+}$ signals from other sources, mitochondrial $\mathrm{Ca}^{2+}$ release in response to $10 \mu \mathrm{M}$ salinomycin was measured under $\mathrm{Ca}^{2+}$-free conditions and after depletion of $\mathrm{ER} \mathrm{Ca}^{2+}$ stores. Under these conditions the response in CGP-treated cells was significantly reduced compared with that in vehicle-treated cells $(12.8 \pm 1.1 \mathrm{nM}, \quad P<0.05,34 / 6$; Figure $2 \mathrm{~d})$. In the presence of extracellular $\mathrm{Ca}^{2+}$, pretreatment with $10 \mu \mathrm{M}$ CGP also resulted in a significantly smaller $\mathrm{Ca}^{2+}$ signal (176 $\pm 41 \mathrm{nM;} P<0.05$ compared with vehicle pretreatment, $34 / 6$; Figure $2 \mathrm{f}$ ). Incubation with $30 \mu \mathrm{M}$ of $\mathrm{SN}-6$, an inhibitor of the NCXs in the plasma membrane, ${ }^{11}$ reduced the response amplitude to $124 \pm 11 \mathrm{nM} \quad(P<0.05,36 / 6$; Figure 2f). Combined treatment with SN-6 and CGP almost completely abrogated the response $(44 \pm 4 \mathrm{nM}, \quad P<0.05,36 / 6$; Figure 2f).
In summary, these data support the hypothesis that increased cytosolic $\mathrm{Ca}^{2+}$ concentrations in response to salinomycin are mediated by NCXs in the plasma membrane and mitochondria, which change to a reverse mode of action owing to cytosolic $\mathrm{Na}^{+}$accumulation.

Salinomycin treatment activates calpain, caspase 12 , caspase 9 and caspase 3 . Increased cytosolic $\mathrm{Ca}^{2+}$ concentrations can induce cell death by various mechanisms: one potential mechanism is the activation of the $\mathrm{Ca}^{2+}$-activated protease calpain in mitochondria, resulting in the release of apoptosis-inducing factor (AIF), which in turn triggers caspase-independent cell death. ${ }^{12}$ In order to investigate this possibility, we visualized AIF in DRGNs treated with a vehicle or salinomycin for $8 \mathrm{~h}$. In either case, AIF showed typical mitochondrial localization and similar staining intensities, which indicates that AIF is not relevant for salinomycin-induced cell death in DRGNs (Figure 3a). a
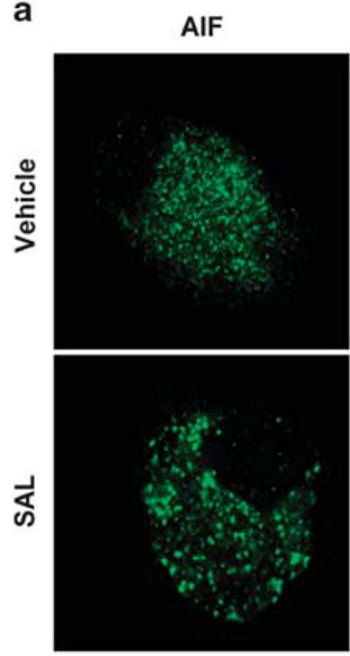

b

b Cytochrome C
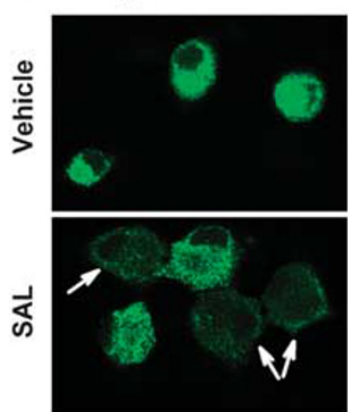

Cytochrome C
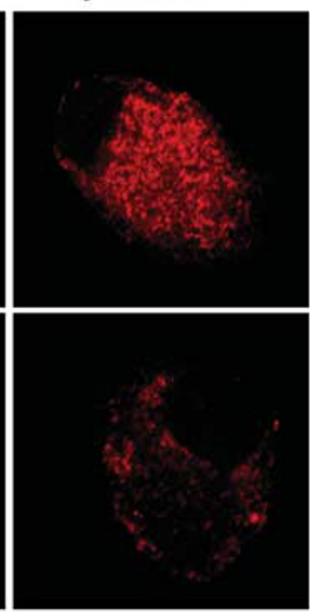

Complex $\mathrm{V}_{\alpha}$
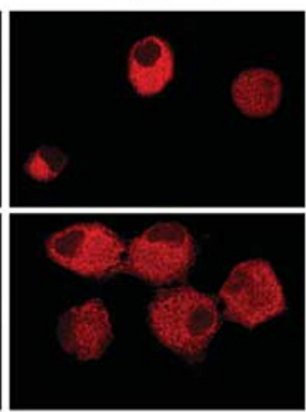

DAPI
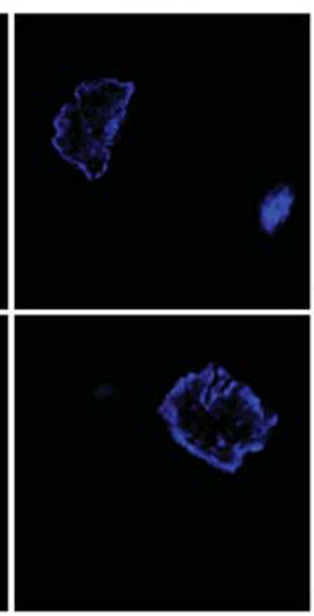

DAPI
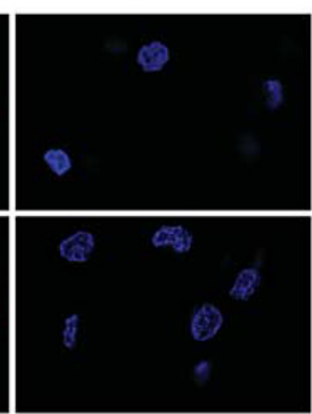

Overlay
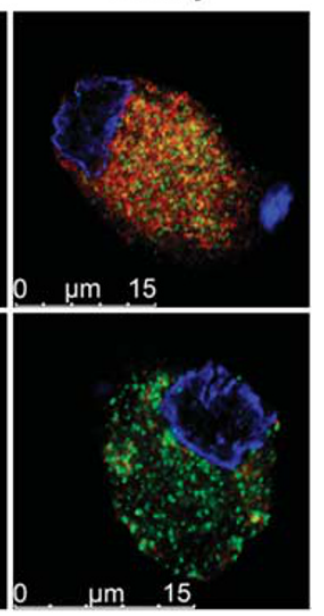

Overlay
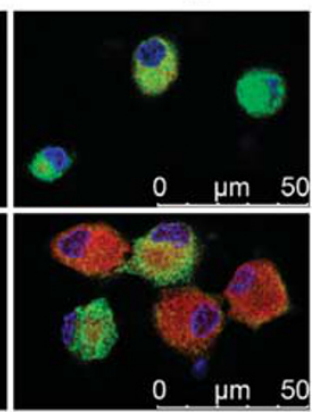

Figure 3 Salinomycin treatment does not alter the localization of AIF but induces the loss of cytochrome $c$ in DRGNs. (a) Representative micrographs of cells treated for $8 \mathrm{~h}$ with vehicle (upper row) or $10 \mu \mathrm{M}$ salinomycin (SAL; lower row). In the first panel AIF was visualized with an Alexa-488-conjugated secondary antibody (green); in the second panel cytochrome $c$ was detected with an Alexa-546-coupled secondary antibody (red); in the third panel DNA was stained with DAPI (blue); and the fourth panel shows an overlay of the previous images. AIF (green) shows similar staining patterns in vehicle- as well as salinomycin-treated cells, whereas the cytochrome $c$ signal (red) is greatly reduced. (b) Representative micrographs of cells treated for $8 \mathrm{~h}$ with vehicle (upper row) or $10 \mu \mathrm{M}$ salinomycin (SAL, lower row). In the first panel cytochrome $c$ was visualized with an FITC-conjugated secondary antibody (green); in the second panel respiratory chain complex V subunit $\alpha$ was detected with an Alexa-633-coupled secondary antibody (red); in the third panel DNA was stained with DAPI (blue); in the fourth panel images were overlaid. Cytochrome $c$ (green) staining is greatly reduced in some of the salinomycin-treated cells (white arrows), whereas staining of respiratory chain complex V subunit $\alpha$ is similar, suggesting release of cytochrome $c$ from mitochondria in these cells 

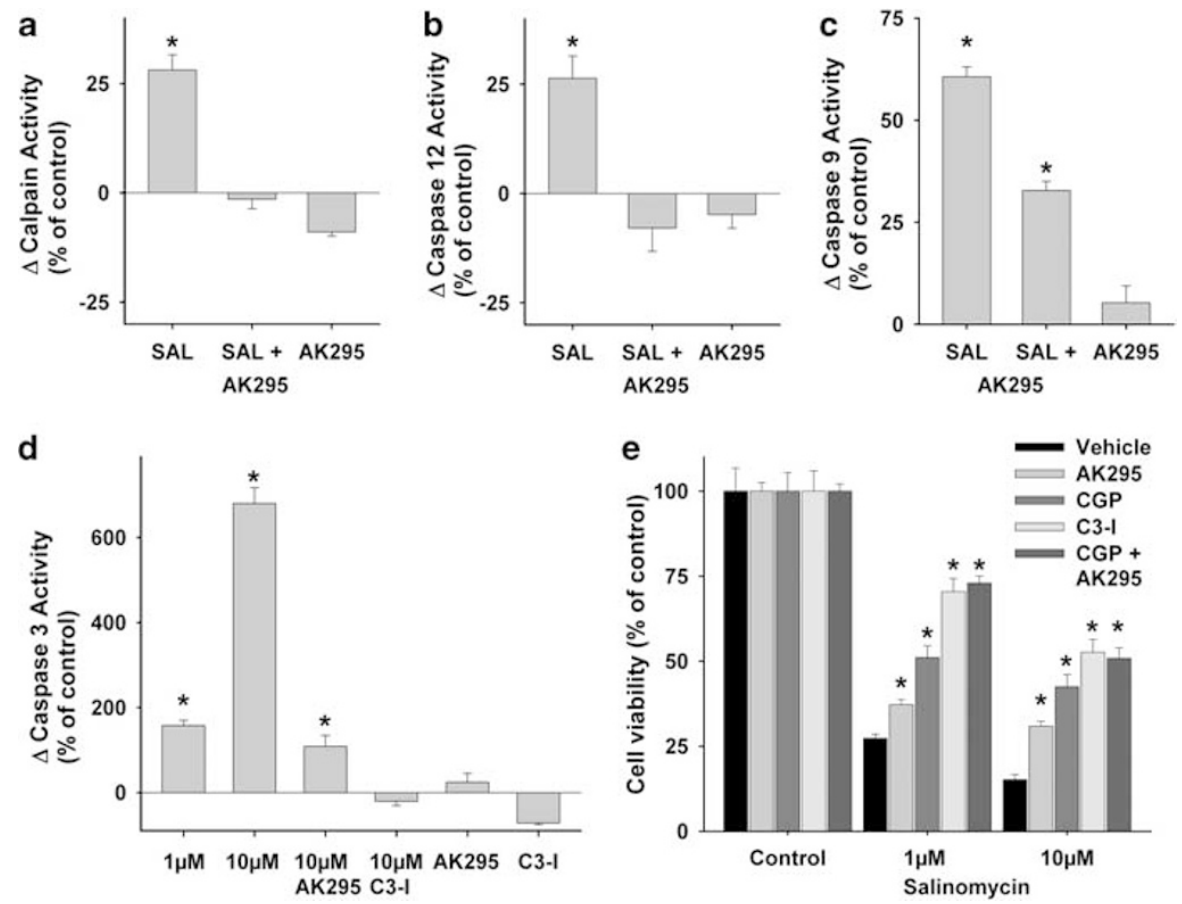

Figure 4 Salinomycin treatment activates calpain, caspase 12, caspase 9 and caspase 3. (a) Treatment of DRGNs with $10 \mu \mathrm{M}$ salinomycin for $8 \mathrm{~h}$ leads to a significant increase in calpain activity. This effect can be prevented with the calpain inhibitor AK295. (b) A similar pattern of activation is observed for caspase 12 activity, which can also be blocked with AK295. Caspase 9 and caspase 3 activity is increased after incubation of DRGNs with $10 \mu \mathrm{M}$ salinomycin for $8 \mathrm{~h}$ (c and d), an effect that is only partly inhibited by the calpain inhibitor AK295. (d) Treatment with the caspase 3 inhibitor C3-I prevents salinomycin-induced caspase 3 activation $(1 \mu \mathrm{M} / 10 \mu \mathrm{M}$ indicates incubation with

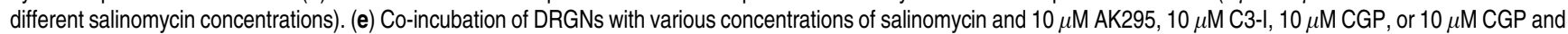
$10 \mu \mathrm{M}$ AK295 for $24 \mathrm{~h}$ significantly improves cell viability. The combination of CGP and AK295 showed comparable effectiveness to the caspase 3 inhibitor (C3-I). ${ }^{*} P<0.05$

Another putative mechanism is the activation of calpain in the cytoplasm, which can cause cellular damage leading to necrosis as well as activation of caspase $12 .{ }^{13}$ In order to explore this possibility, we measured calpain activation $8 \mathrm{~h}$ after treatment with $10 \mu \mathrm{M}$ salinomycin. Calpain activity was significantly higher in salinomycin-treated cells $(128 \pm 4 \%)$ as compared with that in vehicle-treated controls $(100 \pm 3 \%, P<0.001(n=5))$. The calpain inhibitor AK295 has previously been shown to be protective in DRGNs with a deranged intracellular $\mathrm{Ca}^{2+}$ homeostasis. ${ }^{14}$ Incubation with $10 \mu \mathrm{M}$ AK295 prevented the increased calpain activity in salinomycin-treated cells $(99 \pm 2 \% ; P<0.001$ compared with salinomycin treatment $(n=5)$; Figure $4 a)$. By contrast, treatment of cells with AK295 alone did not have a significant effect on the activity of calpain or the studied caspases as compared with the control (Figure 4a-d). Coincubation of DRGNs with $10 \mu \mathrm{M}$ AK295 as well as various concentrations of salinomycin significantly improved cell viability (Figure 4e).

Normalized caspase 12 activity in DRGNs after $8 \mathrm{~h}$ of salinomycin treatment was $126 \pm 5 \%$ as compared with $100 \pm 4 \%$ after vehicle treatment $(n=7 ; P=0.004)$. This effect could be completely inhibited by co-incubation with the calpain inhibitor AK295 (92 $\pm 5 \%$; Figure 4b).

It is known that caspase 12 activates caspase 9, which in turn activates the 'effector' caspase 3 . We were therefore interested to find out whether caspase 9 activity is increased in salinomycin-treated DRGNs. Normalized caspase 9 activity was $161 \pm 2 \%$ in cells treated with $10 \mu \mathrm{M}$ salinomycin for $8 \mathrm{~h}$ as compared with that in vehicle-treated cells $(100 \pm 4 \%, P<0.001(n=5))$. Interestingly, this effect was inhibited only partially by co-incubation with AK295, suggesting additional means of caspase 9 activation (Figure 4c). Similar results were obtained for caspase 3 , which showed marked activation after $8 \mathrm{~h}$ exposure to $10 \mu \mathrm{M}$ salinomycin (780 $\pm 37 \% ; n=5)$ as compared with the vehicle (100 $\pm 16 \%$, $P<0.001(n=5))$. This effect could be inhibited completely by the caspase 3 inhibitor AC-DEVD-CHO (C3-I) at a concentration of $10 \mu \mathrm{M}$, but again only partial inhibition was achieved with AK295 (Figure 4d). Accordingly, treatment of DRGNs with the same caspase 3 inhibitor significantly improved $(P<0.05)$ normalized cell viability to $70 \pm 4 \%(n=9)$ in cells treated with $1 \mu \mathrm{M}$ salinomycin and $53 \pm 4 \%(n=9)$ in cells treated with $10 \mu \mathrm{M}$ salinomycin as compared with that in the control (Figure 4e).

Previous findings from cats suffering from salinomycin intoxication indicated that salinomycin, in addition to axonal damage, also destroys the myelin sheath. ${ }^{5}$ We therefore investigated whether salinomycin in the low micromolar concentration range would impair cell viability in a culture of primary rat Schwann cells. Schwann cells showed a more pronounced reduction in cell viability as compared with DRGNs after exposure to $10 \mu \mathrm{M}$ salinomycin, but were less affected by the lower dose (Figure 5a). Activation of caspase 9 and caspase 3 showed a similar pattern as compared with DRGNs (Figures 5b and c). 

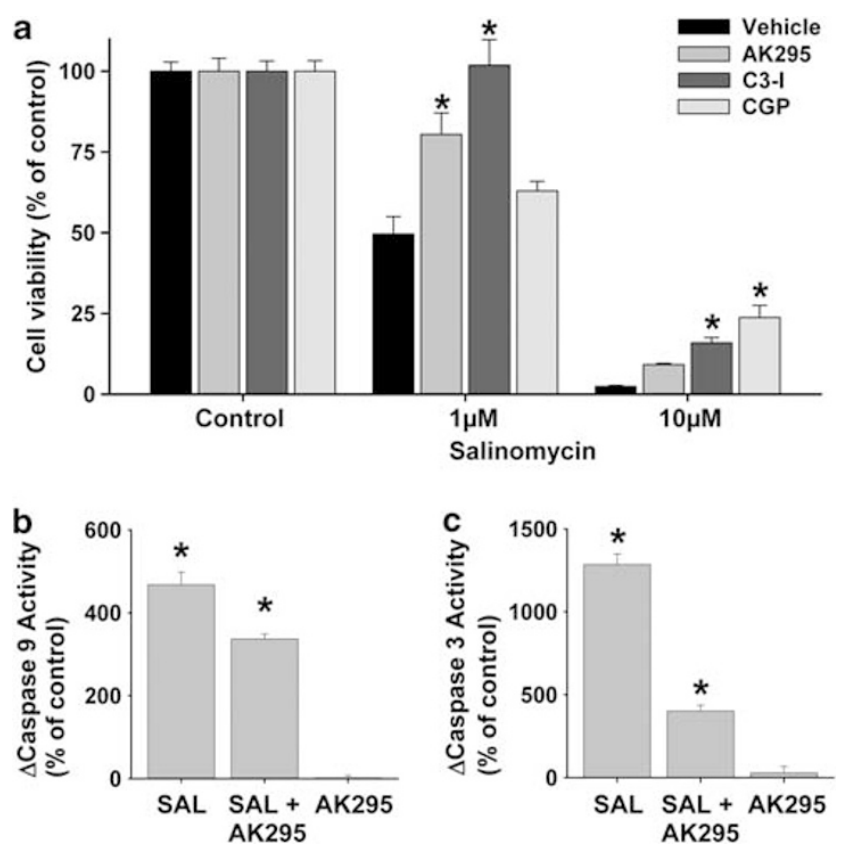

Figure 5 Salinomycin impairs cell viability and activates caspase 9 and caspase 3 in primary Schwann cells. (a) Schwann cells were treated with various concentrations of salinomycin or vehicle (control) for $24 \mathrm{~h}$. Cell viability was measured with a multiplexed assay measuring the activity of the mitochondrial dehydrogenase as well as protease activity released from dead cells. Salinomycin treatment resulted in a marked reduction of cell viability. This effect could be variably influenced by inhibition of mitochondrial NCXs (10 $\mu \mathrm{M}$ CGP), calpain (10 $\mu \mathrm{M}$ AK295) or caspase 3 (10 $\mu \mathrm{M}$ C3-I). (b) Increased caspase 9 and caspase 3 activity is measured after incubation of Schwann cells with $10 \mu \mathrm{M}$ salinomycin for $8 \mathrm{~h}$ (b and c), showing a similar pattern compared to DRGNs. ${ }^{*} P<0.05$

These results suggest that caspase-mediated cell death has an important role in salinomycin-mediated neurotoxicity. Caspase activation appears to be, at least in part, induced by calpain-mediated caspase 12 cleavage, which then triggers the downstream caspases.

Inhibition of the mitochondrial NCXs prevents salinomycin-induced $\Delta \Psi_{\mathrm{m}}$ dissipation and cytochrome $\boldsymbol{c}$ release. It has been reported previously that salinomycin inhibits mitochondrial respiration in isolated mitochondria ${ }^{8}$ and affects the mitochondrial membrane potential $\left(\Delta \Psi_{\mathrm{m}}\right)$ in spermatozoa. ${ }^{15}$ We thus hypothesized that in DRGNs salinomycin might also dissipate $\Delta \Psi_{\mathrm{m}}$, which typically precedes cytochrome $c$ release (reviewed by Green and Reed $\left.{ }^{16}\right)$. Cytochrome $c$ is in turn capable of forming a complex with APAF-1, which leads to the activation of caspase 9. Mitochondrial membrane potential was measured using the cationic fluorescent dye JC-1 by flow cytometry. This dye undergoes a red-green shift of its fluorescence, which correlates with a decrease in the mitochondrial membrane potential. DRGNs treated with $10 \mu \mathrm{M}$ salinomycin for $8 \mathrm{~h}$ showed a marked reduction of red JC-1 fluorescence (Figure 6a) compared with that in vehicle-treated controls. Interestingly, this effect could be inhibited with CGP, an inhibitor of mitochondrial NCXs (Figures 6a and b).
In a next step, we studied cytochrome $c$ release from mitochondria. Cytochrome $c$ was visualized in DRGNs receiving vehicle or salinomycin treatment for $8 \mathrm{~h}$. In salinomycin-treated cells cytochrome $c$ fluorescence was decreased, with many cells showing only a faint signal compared with that in control cells, which suggests that cytochrome $c$ release took place in cells exposed to salinomycin (Figures $3 a$ and b). In order to further characterize this effect, we isolated mitochondrial protein fractions from DRGNs treated for $24 \mathrm{~h}$ with vehicle, $10 \mu \mathrm{M}$ salinomycin, $10 \mu \mathrm{M}$ salinomycin and $10 \mu \mathrm{M}$ CGP, or $10 \mu \mathrm{M}$ CGP alone. Cytochrome $c$ immunoreactivity normalized to the loading control ( $\alpha$-subunit of respiratory chain complex $\mathrm{V})$ showed a significant decrease to $45 \pm 4 \%(n=4)$ in salinomycin-treated cells versus $91 \pm 14 \%$ in salinomycin- and CGP-, and $100 \pm 3 \%$ in vehicle-treated cells $(P<0.05$; Figures $6 \mathrm{c}$ and d). Incubation with CGP alone neither elicited a significant change in $\Delta \Psi_{m}$ nor in cytochrome clevels. These results show a protective effect of CGP on the mitochondrial membrane potential and cytochrome $c$ release in salinomycin-treated cells. We next tested whether inhibition of mitochondrial NCXs would improve cell viability. Co-incubation with CGP improved normalized cell viability to $51 \pm 3 \%(n=9)$ for $1 \mu \mathrm{M}$ salinomycin and $43 \pm 4 \%$ for $10 \mu \mathrm{M}$ salinomycin $(P<0.05$; Figure $4 \mathrm{e})$.

These findings suggest a dual activation mechanism of caspase 9 in salinomycin-treated DRGNs, through calpain and caspase 12 on the one and cytochrome $c$ on the other hand. Indeed, similar levels of cell viability for 1 or $10 \mu \mathrm{M}$ salinomycin were observed in DRGNs treated with C3-I, or those subjected to combined AK295 and CGP treatment, lending support to this hypothesis (Figure 4e).

\section{Discussion}

The aim of this study was to investigate the effects of salinomycin in low micromolar concentrations on the cell viability and ional homeostasis of DRGNs. We observed loss of plasma membrane asymmetry suggestive of early apoptosis after $8 \mathrm{~h}$ and a marked reduction of cell viability after $24 \mathrm{~h}$ of salinomycin treatment. Investigation of the underlying molecular pathomechanism showed that salinomycin mediated an increase of cytosolic $\mathrm{Na}^{+}$and $\mathrm{Ca}^{2+}$ concentrations. This is most likely because of the ionic properties of salinomycin, which have been characterized extensively in previous studies. $^{1}$ Further dissection of the $\mathrm{Ca}^{2+}$ signal showed $\mathrm{Ca}^{2+}$ influx across the plasma membrane as well as release from mitochondrial stores. This effect was mediated by the NCXs in the plasma membrane and mitochondria, which reverse their mode of action in the presence of high cytosolic $\mathrm{Na}^{+}$concentrations. Depletion of $\mathrm{Ca}^{2+}$ stored in the ER leads to a decreased response amplitude compared with that under $\mathrm{Ca}^{2+}$-free conditions alone. This could be because of $\mathrm{Ca}^{2+}$ depletion by prolonged maintenance of cells under $\mathrm{Ca}^{2+}$ buffering conditions. Alternatively, the $\mathrm{Ca}^{2+}$ signal originating in the mitochondria might be amplified through ryanodine receptor-mediated, $\mathrm{Ca}^{2+}$-induced $\mathrm{Ca}^{2+}$ release from the ER. Downstream from these events we observed the activation of the $\mathrm{Ca}^{2+}$-activated protease calpain, which in turn cleaved caspase 12. Caspases are activated in a sequential manner and caspase 12 is upstream from caspase 

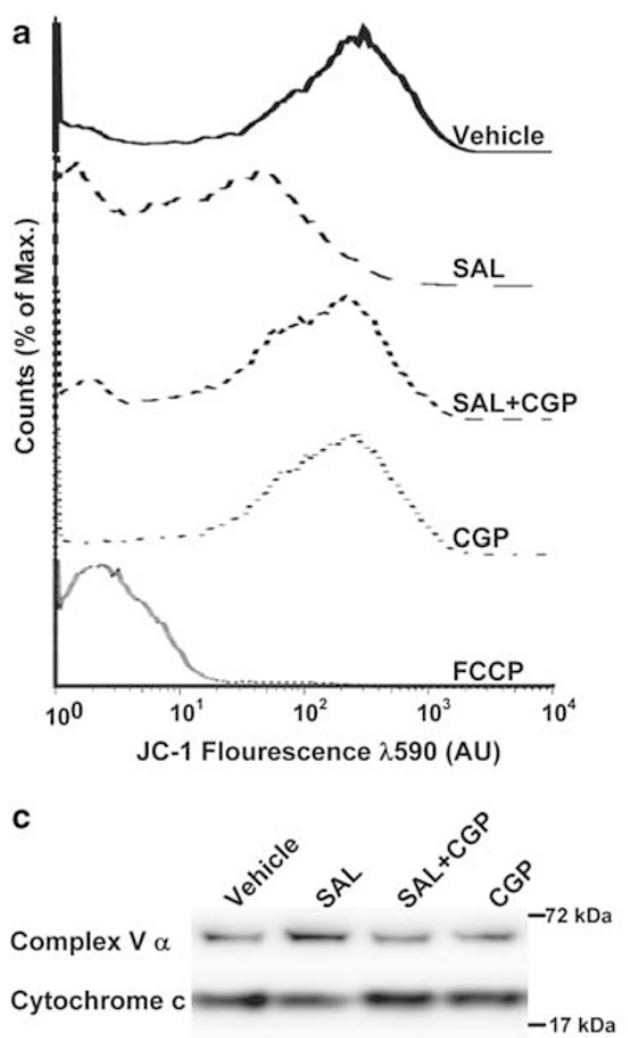

b
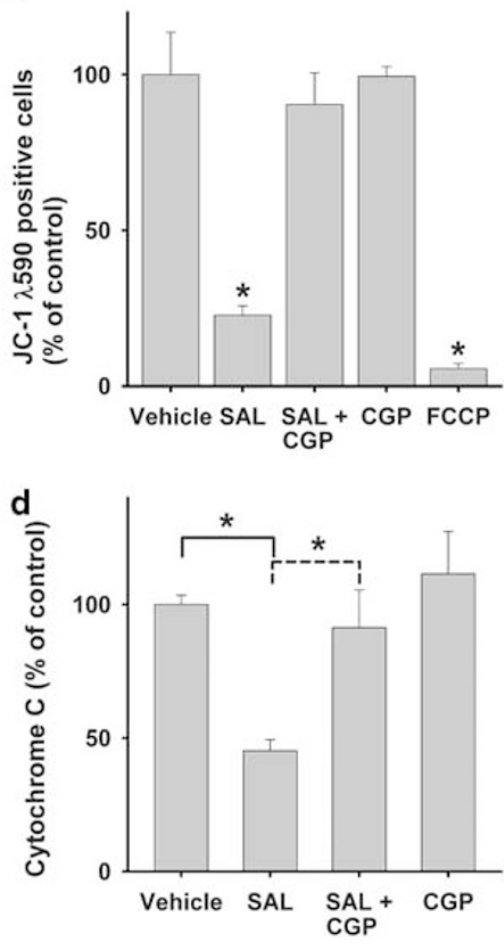

Figure 6 Inhibition of mitochondrial NCXs prevents salinomycin-induced $\Delta \Psi_{\mathrm{m}}$ dissipation and cytochrome $c$ release. (a) Flow cytometric measurement of red JC-1 fluorescence correlates with the mitochondrial membrane potential $\Delta \Psi_{\mathrm{m}}$. DRGNs were treated for $8 \mathrm{~h}$ with vehicle (thick solid line), $10 \mu \mathrm{M}$ salinomycin (SAL, long dashed line), $10 \mu \mathrm{M}$ salinomycin and $10 \mu \mathrm{M} \mathrm{CGP}$, an inhibitor of mitochondrial NCXs (SAL + CGP, short dashed line), $10 \mu \mathrm{M}$ CGP alone (dotted line), or for 30 min with $50 \mu \mathrm{M}$ of the mitochondrial uncoupler FCCP (FCCP, thin line). The data have been presented as normalized histogram. Salinomycin leads to a decreased mitochondrial membrane potential $\Delta \Psi_{\mathrm{m}}$, which can be prevented by co-incubation with CGP. Cells with fluorescence greater than $10^{2}$ (arbitrary units, AU) were classified as JC-1-positive and normalized to control to compare the depolarization induced by salinomycin (b). (c) A representative western blot of the mitochondrial fraction of DRGNs treated for $24 \mathrm{~h}$ with vehicle, $10 \mu \mathrm{M}$ salinomycin (SAL), $10 \mu \mathrm{M}$ salinomycin and $10 \mu \mathrm{M} \mathrm{CGP}$ (SAL + CGP), or $10 \mu \mathrm{M} \mathrm{CGP}$, and probed for cytochrome $c$ and respiratory chain complex V subunit $\alpha$ (loading control). Decreased cytochrome $c$ immunoreactivity is detected after salinomycin treatment. This effect is inhibited by co-incubation with CGP. (d) Normalized cytochrome $c$ immunoreactivity is significantly reduced in salinomycin-treated cells. ${ }^{*} P<0.05$

9 and the 'effector' caspase 3. Whereas inhibition of calpain completely prevented caspase 12 activation, it suppressed caspase 9 and caspase 3 activity only partially, suggesting an alternative pathway for caspase 9 activation.

A well-characterized mechanism of pro-caspase 9 cleavage is through a complex formed by cytochrome $c$ released from mitochondria, dATP and APAF-1. ${ }^{17} \mathrm{We}$ observed that DRGNs treated with salinomycin had a reduced mitochondrial membrane potential and significantly reduced cytochrome $c$ levels in the mitochondrial fraction. Interestingly, inhibition of mitochondrial NCXs prevented loss of mitochondrial membrane potential as well as cytochrome $c$ release, suggesting that in the studied concentration range these effects are mediated by the mitochondrial NCXs and not by direct salinomycin action as observed in isolated mitochondria. $^{8}$ These effects would support a model where caspase 9 is activated by a dual mechanism through both cytochrome $c$ and caspase 12 mediated pathways (Figure 7).

Further support for this model comes from the observation that inhibition of caspase 3 significantly improves cell viability, which allows the conclusion that cell death especially at lower salinomycin concentrations is conferred by caspase-mediated apoptotic cell death. The smaller effect of caspase inhibition on cell survival at higher salinomycin concentrations might be because of a metabolic overload resulting from tonically elevated cytosolic $\mathrm{Na}^{+}$levels. Combined inhibition of calpain and mitochondrial NCXs lead to cell viability comparable with caspase 3 inhibition, supporting dual activation of caspase 9.

Little is known about the effects of salinomycin on the human organism. One published case of salinomycin ingestion in man led to polyneuropathy but also rhabdomyolysis. ${ }^{4}$ As plasma concentrations were not determined, it remains unclear whether damage to striated muscles has to be expected in the dose range of the current study. However, in 1996 food contaminated with salinomycin at a concentration of approximately $30 \mu \mathrm{M}$ led to widespread manifestation of sensorimotor polyneuropathy but not rhabdomyolysis in cats. ${ }^{5,18}$ Therefore, it is likely that that at nanomolar or low micromolar concentrations neurotoxic side effects are predominant. We infer from these anecdotal observations and our data that salinomycin in the studied concentration range bears a significant potential to cause severe peripheral neuropathy in humans, which is highly relevant when this 


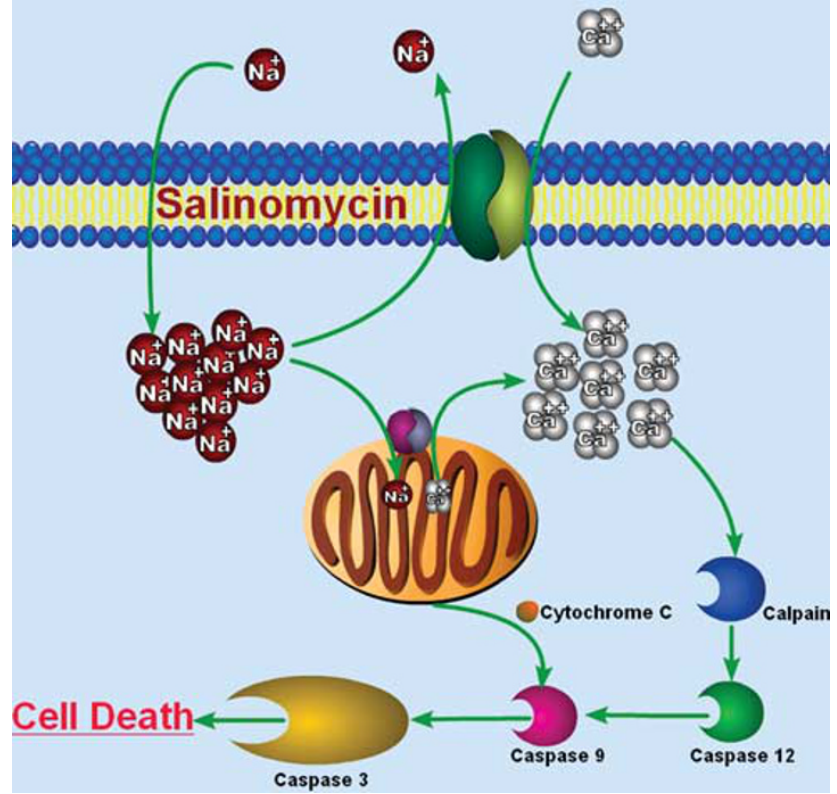

Figure 7 Summary of the observed effects of salinomycin-induced toxicity in DRGNs. Salinomycin leads to an increase of cytosolic $\mathrm{Na}^{+}$concentrations, with a subsequent increase in cytosolic $\mathrm{Ca}^{2+}$, which is mediated by NCXs in the plasma membrane and mitochondria. Elevated intracellular $\mathrm{Ca}^{2+}$ levels activate the protease calpain, which triggers apoptotic cell death by the activation of caspase pathways involving caspase 12 , caspase 9 and caspase 3 . Caspase 9 is additionally activated by cytochrome $c$ released from depolarized mitochondria

toxin is tested as a drug in patients. The preferential occurrence of toxic side effects in the peripheral nervous system may be explained by the observation that P-glycoprotein limits the brain penetration of salinomycin. ${ }^{19}$

Our findings introduce a pathomechanism for salinomycininduced neurotoxicity in the concentration range shown to be effective against CSCs. One central question in this regard is, whether this pathomechanism differs from the mechanism of action against CSCs. Gupta et al., ${ }^{2}$ who identified salinomycin in a high-throughput screen, showed that salinomycin induces a profound change in the gene expression of CSCs. Specifically, they observed reduced expression of genes associated with CSCs and increased expression of cell adhesion proteins such as cadherins. This suggests the conclusion that in CSCs salinomycin leads to terminal epithelial differentiation and subsequent cell-cycle arrest rather than direct cytotoxic effects, which were observed in this study with neuronal cells. How salinomycin induces these changes in gene expression remains to be elucidated; however, the mechanism of action is hypothesized to result from interference with the cellular $\mathrm{K}^{+}$homeostasis. In fact, in line with this notion, $\mathrm{K}^{+}$channels have been shown to be relevant for tumor proliferation and migration (reviewed by $\mathrm{Beug}^{20}$ ). If this holds true, the pathogenesis of neurotoxic side effects would be different from the mode of action against CSCs, enabling neuroprotective strategies without compromise in antitumor activity. Based on our results, a preventive cotreatment with inhibitors of mitochondrial NCXs might allow the use of higher salinomycin concentrations with less neurotoxic side effects, providing a better chance for CSC elimination.
In the context of polyneuropathy research, our observations underline the importance of a deranged $\mathrm{Ca}^{2+}$ homeostasis. We have recently shown that the cytostatic drug paclitaxel induces $\mathrm{Ca}^{2+}$ oscillations in neuronal cells, ${ }^{21}$ which leads to $\mathrm{Ca}^{2+}$-mediated calpain activation. ${ }^{14}$ It was shown previously that calpain inhibition prevents paclitaxel-induced neuropathy in vivo. ${ }^{22}$ Taken together, these findings emphasize the importance of altered intracellular $\mathrm{Ca}^{2+}$ concentrations in the pathogenesis of peripheral neurodegeneration. Our present study further elucidates signal cascades downstream from calpain activation and additionally highlights the role of mitochondrial dysfunction for the induction of cell death in peripheral neurons. Intriguingly, the combination of a disturbed $\mathrm{Ca}^{2+}$ homeostasis and mitochondrial malfunction, although likely initiated by different mechanisms, has also been implied as an important factor in diabetic neuropathy (reviewed by Verkhratsky and Fernyhough ${ }^{23}$ ). This suggests a more general role of these factors in the pathogenesis of polyneuropathy worth further exploration.

We report that exposure of dorsal root ganglia with low micromolar concentrations of salinomycin disturbs the intracellular $\mathrm{Na}^{+}$and $\mathrm{Ca}^{2+}$ homeostasis, resulting in calpain and caspase activation leading to cell death. These findings expand our knowledge of the mechanisms involved in the pathogenesis of salinomycin-induced peripheral neuropathy and provide a mechanism for neuroprotection through inhibition of mitochondrial NCXs. As a result, new strategies for a clinical translation of salinomycin therapy may be developed.

\section{Materials and Methods \\ Cell culture}

$D R G N$ culture. All experimental procedures conformed to institutional guidelines and were approved by an official committee (LaGeSo; T0119/10). DRGNs isolated from rat neonates (P1-3) were digested in 0.28 Wünsch unit collagenase (Liberase DL; Roche, Mannheim, Germany) and separated by gentle trituration. The triturated cells were passed through a 70- $\mu \mathrm{m}$ cell strainer to remove cell clumps, followed by Percoll gradient centrifugation $(1.019 / 1.038 \mathrm{~g} / \mathrm{ml})$ at $1000 \times g$ for $10 \mathrm{~min}$. DRGNs were plated on poly-L-lysine/laminin-coated coverslips and maintained as described previously. ${ }^{14}$

Schwann cell culture. All experimental procedures conformed to institutional guidelines and were approved by an official committee (LaGeSo; T0119/10). Schwann cells were isolated from postnatal rat sciatic nerves (P3) and cultured as described previously. ${ }^{24}$

\section{Live-cell imaging}

Fura-2. Cells were cultured on eight-well Ibidi $\mu$-Slides (Ibidi GmbH, Martinsried, Germany) with and loaded with $5 \mu \mathrm{M}$ Fura-2/AM (Molecular Probes, Invitrogen, Darmstadt, Germany) at $37^{\circ} \mathrm{C}$ for $30 \mathrm{~min}$ in a standard solution containing (in $\mathrm{mM}$ ) $130 \mathrm{NaCl}, 4.7 \mathrm{KCl}, 1 \mathrm{MgSO}_{4}, 1.2 \mathrm{KH}_{2} \mathrm{PO}_{4}, 1.3 \mathrm{CaCl}_{2}, 20 \mathrm{HEPES}$ and 5 glucose $(\mathrm{pH}$ 7.4), and $0.02 \%$ pluronic F-127 (Molecular Probes, Invitrogen). After loading, Fura-2 was allowed to de-esterify for at least $10 \mathrm{~min}$ at room temperature in a standard solution. Cells were monitored using an inverted Olympus IX71 stage equipped with a UPLSAPO X2 $\times 40 / 0.95$ objective (Olympus, Hamburg, Germany). Fluorescence data were acquired using a PC running CellMM software (Olympus) by means of a cooled CCD camera (ORCA; Hamamatsu, Herrsching am Ammersee, Germany). Unless cells were treated with thapsigargin or FCCP, addition of $10 \mu \mathrm{M}$ ionomycin at the end of the experiment was used as an internal control.

Cytosolic free $\mathrm{Ca}^{2+}$ concentration ([$\left.\left[\mathrm{Ca}^{2+}\right]_{\text {int }}\right)$ was calculated as described previously. ${ }^{14}$ Experiments in the absence of $\mathrm{Ca}^{2+}$ were conducted in $\mathrm{Ca}^{2+}$-free HEPES buffer containing (in mM) $130 \mathrm{NaCl}, 4.7 \mathrm{KCl}, 2.3 \mathrm{MgSO}_{4}, 1.2 \mathrm{KH}_{2} \mathrm{PO}_{4}, 10$ EGTA, 20 HEPES and 5 glucose (pH 7.4). All substances were bath-applied. 
SBFI. Cells were cultured on eight-well Ibidi $\mu$-Slides with and loaded with $7 \mu \mathrm{M}$ $\mathrm{SBF} / \mathrm{AM}$ (Molecular Probes, Invitrogen) at $37^{\circ} \mathrm{C}$ for $30 \mathrm{~min}$ in the same standard solution as described above. After loading, SBFI/AM was allowed to de-esterify for at least $10 \mathrm{~min}$ at room temperature in a standard solution. Cells were monitored as described above for Fura-2. The SBFI ratio was calculated from whole-cell fluorescence of background-subtracted images acquired at a wavelength of $340 \mathrm{~nm}$ and $380 \mathrm{~nm}$. Increase in SBFI ratio of each cell was calculated by subtracting the baseline ratio from the stimulated ratio. For comparison the average of this measure was calculated for different treatment conditions.

Assessment of $\Delta \boldsymbol{\Psi}_{\mathrm{m}}$. Cell suspensions with $1.5 \times 10^{5}$ freshly isolated DRGNs were incubated for $8 \mathrm{~h}$ at $37^{\circ} \mathrm{C}$ with vehicle, salinomycin, salinomycin + CGP, CGP or left untreated. Thereafter, cells were loaded with the cationic dye JC-1 at $0.1 \mu \mathrm{M}$ for $30 \mathrm{~min}$, followed by centrifugation for $5 \mathrm{~min}$ at $300 \times g$ and resuspension in buffer containing $1 \%$ BSA in phosphate-buffered saline (PBS) ( $\mathrm{pH}$ 7.4). The untreated samples were additionally incubated with $50 \mu \mathrm{M} \mathrm{FCCP}$ as a depolarized control. Analysis was performed with a 'FACSCalibur' (BD Biosciences, Heidelberg, Germany) flow cytometer, where JC1 was excited by an argon laser $(488 \mathrm{~nm})$ and green $(530 / 30 \mathrm{~nm})$ as well as red fluorescence $(585 / 42 \mathrm{~nm})$ was detected simultaneously. Data were analyzed using the FlowJo software (Tree Star Inc., Ashland, OR, USA).

\section{Cell viability assays}

Annexin-V labeling. Cells were incubated on a 48-well plate for $8 \mathrm{~h}$ with vehicle or salinomycin. Annexin-V labeling was performed and analyzed using EGFPconjugated annexin-V (MBL, Woburn, MA, USA) according to the manufacturer's protocol. Annexin-V-EGFP staining was visualized using an inverted Leica DM3000 epiflourescence microscope (Leica, Wetzlar, Germany). Two images were taken per well and the number of annexin-V-positive cells was counted.

CytoTox-Fluor cytotoxicity assay. This assay measures a distinct protease activity associated with cytotoxicity and uses a fluorogenic peptide substrate (bis-alanyl-alanyl-phenylanlanyl-rhodamine 110 (bis-AAF-R110)) to assess protease activity that has been released from cells that have lost membrane integrity (Promega, Mannheim, Germany). This assay was performed prior to MTT assay, by pipetting $50 \mu \mathrm{l}$ of supernatant from treated cells on a 96-well plate, followed by addition of $50 \mu \mathrm{l}$ of substrate and incubation for $1 \mathrm{~h}$ at $37^{\circ} \mathrm{C}$ Fluorescence was measured using a fluorescence microplate reader (CytoFluor; Perseptive Biosystems, Carlsbad, CA, USA), using $480 \pm 20 \mathrm{~nm}$ as the excitation and $525 \pm 20 \mathrm{~nm}$ as the emission wavelength. The values were background-subtracted and a compound measure for cell viability was calculated as described below.

MTT assay. Metabolic integrity of cells was assessed using MTT as described previously. ${ }^{25}$ The MTT assay correlates with the number of live cells, whereas the CytoTox-Fluor assay correlates with the number of dead cells. In order to increase sensitivity, both assays where combined by dividing background-subtracted MTT absorbance values by the background-subtracted values of the cytotoxicity assay. The values were then normalized to control.

Cell fractionation and western blot analysis. Purified cytosolic, nuclear and mitochondrial fractions were obtained using the Cell fractionation kit HT (Mitosciences, Eugene, OR, USA) following the manufacturer's protocol. Immunoblotting was performed as described previously. ${ }^{26}$ The antibodies used were as follows: anti-cytochrome $c$ and anti-complex- $\mathrm{V}-\alpha$ (both from Mitosciences). Blots were quantified by scanning densitometry using the ImageJ software (National Institutes of Health, Bethesda, MD, USA), by normalizing the cytochrome clevels in the mitochondrial fraction to the complex-V- $\alpha$ loading control. All western blot experiments were performed using three independent cultures unless stated otherwise.

\section{Protease assays}

Calpain activity assay. Calpain activity was assessed as described previously. ${ }^{27}$ Cells were grown in 96 -well plates and incubated for $30 \mathrm{~min}$ with inhibitors as well as the Suc-LLVY-aminoluciferin calpain substrate at $20 \mu \mathrm{M}$, followed by treatment with salinomycin or vehicle (DMSO). After $8 \mathrm{~h}$ of treatment, cells were lysed with $0.9 \%$ Triton X-100 in presence of $0.1 \mathrm{mM} \mathrm{MDL}-28170$, a potent inhibitor of calpain-1 and calpain-2. After addition of the CalpainGLO luciferase detection reagent from the Calpain Glo protease assay (Promega) luminescence was detected using a Berthold Centro LB 960 microplate reader (Berthold, Schöneiche/Berlin, Germany).
Caspase-3/7 assay. Caspase-3 activity was measured by the Apo-ONE homogeneous caspase- $3 / 7$ assay (Promega) according to the manufacturer's protocol.

Caspase- 9 assay. Caspase- 9 activity was measured using the Caspase-Glo 9 assay system (Promega) as described in the manufacturer's protocol.

Caspase-12 assay. Caspase-12 activity was measured with a fluorometric caspase-12 assay kit (Promokine; Promocell, Heidelberg, Germany) according to manufacturer's instructions.

Immunocytochemistry of DRGNs. DRGNs were cultivated on poly-Llysine- and laminin-coated coverslips. After fixation with $4 \%$ paraformaldehyde in PBS, cells were permeabilized with $0.1 \%$ Triton X-100. Unspecific binding sites were blocked with $10 \%$ normal goat serum (NGS) and proteins of interest were identified with a primary antibody diluted in $10 \%$ NGS at $4{ }^{\circ} \mathrm{C}$ overnight. In each experiment a negative control without a primary antibody was included. The primary antibodies were detected with a fluorophore coupled secondary antibody and mounted with the ProLong Gold antifade reagent with DAPI (Molecular Probes, Invitrogen). The specimens were visualized with a Leica TCS SPE upright confocal microscope. The antibodies used were as follows: Primary antibodiesanti-cytochrome $c$ and anti-complex-V- $\alpha$ (both from Mitosciences), and anti-AIF (Epitomics, Burlingame, CA, USA); secondary antibodies-anti-mouse $\operatorname{lgG}_{22}-\mathrm{FITC}$ (Mitosciences); anti-mouse $\operatorname{lgG}_{2 b}$-Alexa-633, anti-mouse IgG-Alexa-546 and anti-rabbit IgG-Alexa-488 (all from Molecular Probes, Invitrogen).

Statistical analysis. Data are expressed as the means \pm S.E.M. or as representative traces. $(n / M)$ describes the number of cells studied $(n)$ in $(M)$ independent cultures. Statistical analysis of the differences between multiple groups was performed by one-way ANOVA and for two groups by $t$-test (SigmaStat; Systat, Richmond, CA, USA). $P<0.05$ was considered statistically significant.

\section{Conflict of Interest}

The authors declare no conflict of interest.

Acknowledgements. We thank Christoph Harms for the opportunity to conduct $\mathrm{Ca}^{2+}$-imaging experiments and Barbara Ehrlich for thoughtful discussions and comments on the paper. The research leading to these results received funding from the Federal Ministry of Education and Research through the grant 01 EO 0801 from the Center for Stroke Research Berlin, the Volkswagen Foundation (Lichtenberg program) and the German Research Council DFG (NeuroCure).

1. Mitani M, Yamanishi T, Miyazaki Y. Salinomycin: a new monovalent cation ionophore. Biochem Biophys Res Commun 1975; 66: 1231-1236.

2. Gupta PB, Onder TT, Jiang G, Tao K, Kuperwasser C, Weinberg RA et al. Identification of selective inhibitors of cancer stem cells by high-throughput screening. Cell 2009; 138: 645-659

3. Kim JH, Chae M, Kim WK, Kim YJ, Kang HS, Kim HS et al. Salinomycin sensitizes cancer cells to the effects of doxorubicin and etoposide treatment by increasing DNA damage and reducing p21 protein. Br J Pharmacol 2011; 162: 773-784.

4. Story $\mathrm{P}$, Doube A. A case of human poisoning by salinomycin, an agricultural antibiotic. $N Z$ Med J 2004; 117: U799.

5. van der Linde-Sipman JS, van den Ingh TS, van nes JJ, Verhagen $\mathrm{H}$, Kersten JG, Beynen $\mathrm{AC}$ et al. Salinomycin-induced polyneuropathy in cats: morphologic and epidemiologic data. Vet Pathol 1999; 36: 152-156.

6. Novilla MN, Owen NV, Todd GC. The comparative toxicology of narasin in laboratory animals. Vet Hum Toxicol 1994; 36: 318-323.

7. Lattanzio Jr FA, Pressman BC. Alterations in intracellular calcium activity and contractility of isolated perfused rabbit hearts by ionophores and adrenergic agents. Biochem Biophys Res Commun 1986; 139: 816-821.

8. Mitani M, Yamanishi T, Miyazaki Y, Otake N. Salinomycin effects on mitochondrial ion translocation and respiration. Antimicrob Agents Chemother 1976; 9: 655-660.

9. Galluzzi L, Aaronson SA, Abrams J, Alnemri ES, Andrews DW, Baehrecke EH et al. Guidelines for the use and interpretation of assays for monitoring cell death in higher eukaryotes. Cell Death Differ 2009; 16: 1093-1107.

10. Cox DA, Conforti L, Sperelakis N, Matlib MA. Selectivity of inhibition of $\mathrm{Na}(+)-\mathrm{Ca} 2+$ exchange of heart mitochondria by benzothiazepine CGP-37157. J Cardiovasc Pharmaco 1993; 21: 595-599. 
11. Imamoto $T$, Inoue $Y$, Ito $\mathrm{K}$, Sakaue $\mathrm{T}$, Kita S, Katsuragi T. The exchanger inhibitory peptide region-dependent inhibition of $\mathrm{Na}+/ \mathrm{Ca} 2+$ exchange by $\mathrm{SN}-6$ [2-[4-(4-nitrobenzyloxy) benzyl]thiazolidine-4-carboxylic acid ethyl ester], a novel benzyloxyphenyl derivative. $\mathrm{Mol}$ Pharmacol 2004; 66: 45-55.

12. Norberg E, Gogvadze V, Ott M, Horn M, Uhlen P, Orrenius $S$ et al. An increase in intracellular $\mathrm{Ca} 2+$ is required for the activation of mitochondrial calpain to release AIF during cell death. Cell Death Differ 2008; 15: 1857-1864.

13. Nakagawa T, Yuan J. Cross-talk between two cysteine protease families. Activation of caspase-12 by calpain in apoptosis. J Cell Biol 2000; 150: 887-894.

14. Boehmerle W, Zhang K, Sivula M, Heidrich FM, Lee Y, Jordt SE et al. Chronic exposure to paclitaxel diminishes phosphoinositide signaling by calpain mediated NCS-1 degradation in neuronal cells. Proc Natl Acad Sci USA 2007; 104: 11103-11108.

15. Hoornstra D, Andersson MA, Mikkola R, Salkinoja-Salonen MS. A new method for in vitro detection of microbially produced mitochondrial toxins. Toxicol In Vitro 2003; 17: 745-751.

16. Green DR, Reed JC. Mitochondria and apoptosis. Science 1998; 281: 1309-1312.

17. Pop C, Timmer J, Sperandio S, Salvesen GS. The apoptosome activates caspase-9 by dimerization. Mol Cell 2006; 22: 269-275.

18. Pakozdy A, Challande-Kathman I, Doherr M, Cizinauskas S, Wheeler SJ, Oevermann A et al. Retrospective study of salinomycin toxicosis in 66 cats. Vet Med Int 2010; 2010: 147142.

19. Lagas JS, Sparidans RW, van Waterschoot RA, Wagenaar E, Beijnen JH, Schinkel AH. P-glycoprotein limits oral availability, brain penetration, and toxicity of an anionic drug, the antibiotic salinomycin. Antimicrob Agents Chemother 2008; 52: 1034-1039.

20. Beug H. Breast cancer stem cells: eradication by differentiation therapy? Cell 2009; 138 : 623-625.

21. Boehmerle W, Splittgerber U, Lazarus MB, McKenzie KM, Johnston DG, Austin DJ et al. Paclitaxel induces calcium oscillations via an inositol 1,4,5-trisphosphate receptor and neuronal calcium sensor 1-dependent mechanism. Proc Natl Acad Sci USA 2006; 103 18356-18361.

22. Wang MS, Davis AA, Culver DG, Wang Q, Powers JC, Glass JD. Calpain inhibition protects against Taxol-induced sensory neuropathy. Brain 2004; 127 (Part 3): 671-679.

23. Verkhratsky A, Fernyhough P. Mitochondrial malfunction and $\mathrm{Ca} 2+$ dyshomeostasis drive neuronal pathology in diabetes. Cell Calcium 2008; 44: 112-122.

24. Wei $Y$, Zhou J, Zheng Z, Wang A, Ao Q, Gong $Y$ et al. An improved method for isolating Schwann cells from postnatal rat sciatic nerves. Cell Tissue Res 2009; 337 361-369.

25. Capela JP, Ruscher K, Lautenschlager M, Freyer D, Dirnagl U, Gaio AR et al. Ecstasyinduced cell death in cortical neuronal cultures is serotonin 2A-receptor-dependent and potentiated under hyperthermia. Neuroscience 2006; 139: 1069-1081.

26. Schlecker C, Boehmerle W, Jeromin A, DeGray B, Varshney A, Sharma $Y$ et al. Neuronal calcium sensor-1 enhancement of InsP3 receptor activity is inhibited by therapeutic levels of lithium. J Clin Invest 2006; 116: 1668-1674.

27. Seyb KI, Schuman ER, Ni J, Huang MM, Michaelis ML, Glicksman MA. Identification of small molecule inhibitors of beta-amyloid cytotoxicity through a cell-based high-throughput screening platform. J Biomol Screen 2008; 13: 870-878.

Cell Death and Disease is an open-access journal published by Nature Publishing Group. This work is licensed under the Creative Commons Attribution-Noncommercial-No Derivative Works 3.0 Unported License. To view a copy of this license, visit http://creativecommons.org/licenses/by-nc-nd/3.0/ 